\title{
Breaking of four-fold lattice symmetry in a model for pnictide superconductors
}

\author{
M Daghofer and A Fischer \\ IFW Dresden, P.O. Box 2701 16, D-01171 Dresden, Germany \\ E-mail: m.daghofer@ifw-dresden.de
}

\begin{abstract}
We investigate the interplay of onsite Coulomb repulsion and various mechanisms breaking the fourfold lattice symmetry in a three-band model for the iron planes of iron-based superconductors. Using cluster-perturbation theory allows us to locally break the symmetry between the $x$ - and $y$-directions without imposing long-range magnetic order. Previously investigated anisotropic magnetic couplings are compared to an orbital-ordering field and anisotropic hoppings. We find that all three mechanisms for a broken rotational symmetry lead to similar signatures once onsite interactions are strong enough to bring the system close to a spin-density wave. The band distortions near the Fermi level are independent of differences between the total densities found in $x z$ and $y z$ orbitals.
\end{abstract}

PACS numbers: 74.70.Xa,74.25.Jb,71.27.+a,71.10.Fd

\section{Introduction}

Like cuprate superconductors, many of their iron-based cousins have an antiferromagnetic (AFM) phase in the phase diagram near the superconducting phase [1, 2. As phonons are moreover not believed to be strong enough to explain the relatively high transition temperatures of pnictides [3, the AFM interactions have attracted large interest [4. Apart from the metallic character of the spin-density-wave (SDW) phase in pnictides - as opposed to the Mott insulator in undoped cuprates - a second difference is that the SDW in pnictides breaks the four-fold symmetry of the iron-arsenic planes down to a two-fold symmetry.

This breaking of the four-fold lattice symmetry is seen in the conductivity [5, 6] and in angle-resolved photo-emission spectroscopy (ARPES) [7, 8, 9, 10] even at temperatures above the onset of magnetic long-range order. While there is a structural phase transition at slightly higher temperature and while the in-plane lattice constants thus break the rotational lattice symmetry [11, the effects in experiments appear stronger than can be explained by slightly different lattice constants. Additional symmetry-breaking of the electronic degrees of freedom has thus been suggested to be involved, especially (i) a breaking of the orbital symmetry, i.e., a lifting of the degeneracy of the $x z$ and $y z$ orbitals [12, 13, 14, and (ii) a nematic phase of the spin degree of freedom [15, 16]. In the latter scenario, magnetic correlations already select the preferred ordering vector, e.g., $(\pi, 0)$ over the equivalent $(0, \pi)$, but do not yet establish long-range magnetic order [17, 18, 16, 19]. A related picture involves clusters with short-range magnetic order whose AFM direction is pinned by the lattice anisotropy [20]. 
One problem in deciding between the scenarios is precisely that they all break the same symmetry, which implies that as soon as one of the breaks rotational symmetry, the symmetry in the other degrees of freedom will be broken as well. Lattice [21, 22, 19] and orbital 12, 16, degrees of freedom thus strongly interact with the spin. Nevertheless, identifying the signatures of various modes of symmetry breaking may help to elucidate the most important effects. We calculate here the spectral density of the three-orbital model, see Sec. 2, where the four-fold rotational lattice symmetry is broken by (i) orbital order, (ii) anisotropic hoppings and (iii) short-range AFM interactions. In order to be able to keep the magnetic interactions short-range and in order to include onsite Coulomb interactions, we employ clusterperturbation theory, extending an earlier study [23] of anisotropy driven by shortrange AFM and ferromagnetic (FM) interactions. Anisotropic magnetic interactions can distort the spectral density in agreement with ARPES both in otherwise noninteracting three- and four-band models and for a regime close to the SDW phase [23, orbital order in the non-interacting model was discussed in [14. Here, we compare the two scenarios near the SDW in more detail and also compare them to symmetry breaking via anisotropic hoppings. While distortions induced by the latter cannot even qualitatively be reconciled with ARPES for the noninteracting model, we are going to see that results become more realistic near the SDW transition.

\section{Model and Method}

As we want to use exact diagonalization to solve a fully interacting model on a small four-site cluster, we have to restrict the model to at most three bands. We use a variant [23] of the three-band model proposed in [24] that gives a better fit of the Fermi surface. The unit cell of the Fe-As plane contains two iron and two arsenic ions, however, an internal symmetry of this unit cell allows us to write the Hamiltonian in terms of a one-iron unit cell [25, 26] as long as we consider isolated planes, as is done here. In momentum space, the tight-binding Hamiltonian can then be written in terms of a pseudo-crystal momentum $\tilde{\mathbf{k}}$, which is defined as $\tilde{\mathbf{k}}=\mathbf{k}$ for the $x z / y z$ orbitals and $\tilde{\mathbf{k}}=\mathbf{k}+(\pi, \pi)$ for the $x y$ orbital and which is taken from the enlarged Brillouin zone corresponding to a one-iron unit cell. When translating the spectral density $A(\mathbf{k}, \omega)$ from pseudo-crystal momentum $\tilde{\mathbf{k}}$ to 'lab-space' momentum $\mathbf{k}$, spectral weight of $x y$ character is shifted by $(\pi, \pi)$ with respect to $x z$ and $y z$ weight [27, 14, 23].

The $\tilde{\mathbf{k}}$-dependent Hamiltonian in orbital space can then be written as

$$
H_{\mathrm{TB}}(\tilde{\mathbf{k}})=\sum_{\tilde{\mathbf{k}}, \sigma, \mu, \nu} T^{\mu, \nu}(\tilde{\mathbf{k}}) d_{\tilde{\mathbf{k}}, \mu, \sigma}^{\dagger} d_{\tilde{\mathbf{k}}, \nu, \sigma},
$$

where $d_{\tilde{\mathbf{k}}, \nu, \sigma}\left(d_{\tilde{\mathbf{k}}, \nu, \sigma}^{\dagger}\right)$ annihilates (creates) an electron with pseudo-crystal momentum $\tilde{\mathbf{k}}$ and spin $\sigma$ in orbital $\nu$. The orbital indices $\nu, \mu=1,2,3$ refer to the $x z, y z$ and $x y$ states of the iron $3 d$ manifold, respectively. The $T^{\mu, \nu}(\tilde{\mathbf{k}})=T^{\mu, \nu}\left(k_{x}, k_{y}\right)$ defining the hoppings are given by

$$
\begin{aligned}
T^{11 / 22}= & 2 t_{2 / 1} \cos k_{x}+2 t_{1 / 2} \cos k_{y} \\
& +4 t_{3} \cos k_{x} \cos k_{y} \\
& \pm 2 t_{11}\left(\cos 2 k_{x}-\cos 2 k_{y}\right) \\
& +4 t_{12} \cos 2 k_{x} \cos 2 k_{y} \\
T^{33}= & \Delta_{x y}+2 t_{5}\left(\cos k_{x}+\cos k_{y}\right)
\end{aligned}
$$




$$
\begin{aligned}
&+4 t_{6} \cos k_{x} \cos k_{y}+2 t_{9}\left(\cos 2 k_{x}+\cos 2 k_{y}\right) \\
&+4 t_{10}\left(\cos 2 k_{x} \cos k_{y}+\cos k_{x} \cos 2 k_{y}\right), \\
& T^{12}= T^{21}=4 t_{4} \sin k_{x} \sin k_{y}, \\
& T^{13}=\bar{T}^{31}=2 i t_{7} \sin k_{x}+4 i t_{8} \sin k_{x} \cos k_{y}, \\
& T^{23}=\bar{T}^{32}=2 i t_{7} \sin k_{y}+4 i t_{8} \sin k_{y} \cos k_{x},
\end{aligned}
$$

where a bar denotes the complex conjugate. Hopping parameters are the same as in [23: $t_{1}=-0.08, t_{2}=0.1825, t_{3}=0.08375, t_{4}=-0.03, t_{5}=0.15, t_{6}=0.15$, $t_{7}=-0.12, t_{8}=-t_{7} / 2=0.06, t_{10}=-0.024, t_{11}=-0.01, t_{12}=0.0275, \Delta_{x y}=0.75$. The chemical potential $\mu$ depends on the interaction terms and is chosen to fix the filling at 4 electrons per site, for non-interacting electrons, we find $\mu=0.47$. All energies are given in eV. These bands are only an approximation and a three-band model may not be detailed enough to capture material-dependent properties.

We also include the onsite Coulomb interactions including Hund's rule coupling and pair hopping [28, 29. While the couplings for the $x z / y z$ doublet can in principle differ from the ones involving the $x y$ orbital, we choose them here to be the same and employ the symmetric relations $U=U^{\prime}+2 J$ for simplicity.

$$
\begin{aligned}
H_{\mathrm{int}}= & U \sum_{\mathbf{i}, \alpha} n_{\mathbf{i}, \alpha, \uparrow} n_{\mathbf{i}, \alpha, \downarrow}+\left(U^{\prime}-J / 2\right) \sum_{\mathbf{i}, \alpha<\beta} n_{\mathbf{i}, \alpha} n_{\mathbf{i}, \beta} \\
& -2 J \sum_{\mathbf{i}, \alpha<\beta} \mathbf{S}_{\mathbf{i} \alpha} \cdot \mathbf{S}_{\mathbf{i} \beta} \\
& +J \sum_{\mathbf{i}, \alpha<\beta}\left(d_{\mathbf{i}, \alpha, \uparrow}^{\dagger} d_{\mathbf{i}, \alpha, \downarrow}^{\dagger} d_{\mathbf{i}, \beta, \downarrow} d_{\mathbf{i}, \beta, \uparrow}+\text { h.c. }\right),
\end{aligned}
$$

where $\alpha, \beta$ denote the orbital and $\mathbf{S}_{\mathbf{i}, \alpha}\left(n_{\mathbf{i}, \alpha}\right)$ is the spin (electronic density) in orbital $\alpha$ at site $\mathbf{i}$. The electron-spin operators are given as usual by $\mathbf{S}_{\mathbf{i} \nu}=\frac{1}{2} \sum_{s s^{\prime}} d_{\mathbf{i} \nu s}^{\dagger} \boldsymbol{\sigma}_{s s^{\prime}} d_{\mathbf{i} \nu s^{\prime}}$, where $\boldsymbol{\sigma}=\left(\sigma^{x}, \sigma^{y}, \sigma^{z}\right)$ is the vector of Pauli matrices. We choose here $U=1.02 \mathrm{eV}$ and $J=U / 4$, because the system is then very close to the SDW transition. The interacting system has been found to be very susceptible to magnetic interactions breaking rotational symmetry [23, and we thus concentrate on this regime.

Similar to the approach chosen in [23, we explicitly break rotational symmetry by Heisenberg couplings that act only locally within the small cluster directly solved with exact diagonalization (see method below). Extending the analysis in [23], the coupling $J_{x}$ along the $x$-direction and $J_{y}$ along $y$ can have different magnitudes and the same or different signs:

$$
H_{\text {Heis }}=J_{x} \sum_{\substack{\langle i, j\rangle \| x \\ \mu \nu}} \mathbf{S}_{\mathbf{i}, \mu} \cdot \mathbf{S}_{\mathbf{j}, \nu}+J_{y} \sum_{\substack{\langle\mathrm{i}, \mathrm{j}\rangle \| y \\ \mu \nu}} \mathbf{S}_{\mathbf{i}, \mu} \cdot \mathbf{S}_{\mathbf{j}, \nu}
$$

where $\mu, \nu$ denote orbitals and $\langle\mathbf{i}, \mathbf{j}\rangle \| x / y$ nearest-neighbour (NN) bonds along the $x$ and $y$ directions. For $J_{x / y}>0$, the coupling is AFM.

We compare this magnetic symmetry breaking addressing the anisotropy of the magnetic state to a an orbital symmetry breaking addressing the symmetry between the $x z$ and $y z$ orbitals. The latter is implemented as a difference in onsite energy

$$
H_{\text {orb }}=\Delta \sum_{\mathbf{i}}\left(n_{\mathbf{i}, y z}-n_{\mathbf{i}, x z}\right)
$$

where $\Delta>0$ favours occupation of the $x z$ orbital, as proposed as an explanation of the anisotropic spectral density [14. Finally, as a third way to induce an anisotropy, we make hoppings along one lattice direction five to ten percent larger. 


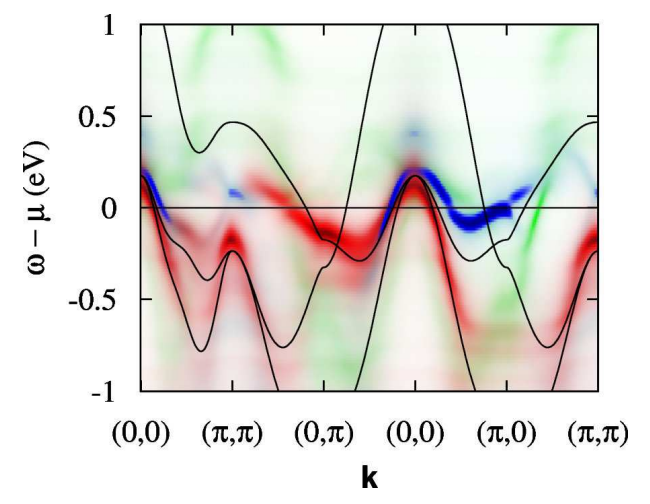

Figure 1. Spectral density $A(\mathbf{k}, \omega)$ for onsite interactions near the SDW transition $(U=1.02 \mathrm{eV}, J=U / 4)$ and a phenomenological field $\Delta=0.1 \mathrm{eV}$, see (9), breaking the orbital $x z / y z$ symmetry and favouring the $x z$ orbital. Solid lines give the noninteracting bands in terms of the pseudo-crystal momentum $\tilde{\mathbf{k}}$; shading gives the spectral weight of the interacting system for 'lab-space' momentum $\mathbf{k}, x y$ weight is thus shifted by $(\pi, \pi)$ with respect to $\tilde{\mathbf{k}}$. In the online colour figure, red, blue and green shading illustrate spectral weight in the $x z, y z$, and $x y$ orbitals respectively.

Following [23, this Hamiltonian is treated with the variational cluster approach. This method allows to include correlations within a small cluster (4 sites for the three-orbital model discussed here), which is solved almost exactly with Lanczos exact diagonalization. Hopping between the clusters is then included as a perturbation 30 , 31. Long-range order can be treated by optimizing the grand potential with respect to fictitious ordering fields [32, 33, the SDW state of a two-band model for pnictides has been studied with this approach [34, 35. In fact, all parameters of the one-particle part of the Hamiltonian can in principle be optimized, we found here that optimizing an overall fictitious chemical potential is necessary near the SDW transition to obtain a stable solution.

\section{Results}

It has been shown that both orbital ordering 14 and anisotropic magnetic correlations 23. can reproduce the band distortions in a manner broadly consistent with ARPES, i.e., the $y z$ states at $X=(\pi, 0)$ move to higher energies than the $x z$ states at $Y=(0, \pi)$. Differences between these two ways of breaking rotational lattice symmetry mostly affect states around $\Gamma=(0,0)$, where an orbital energy splitting induces stronger distortions than the magnetic fluctuations in the absence of onsite interactions 23. As can be seen by comparing figures 1 and 2 this remains true when onsite interactions push the system closer to the SDW transition. One may also note that despite the sizable onsite interactions, one still needs an orbital energy splitting $\Delta=0.1 \mathrm{eV}$ to induce the band anisotropy between $X$ and $Y$ as seen in figure 1 The anisotropy in the final band structure is here not even extreme, with the $y z$ states not quite reaching the Fermi level, and $\Delta$ of a similar order of magnitude induces comparable distortions in non-interacting bands [14, 23]. This is in contrast to shortrange magnetic couplings that become more effective at distorting the bands when onsite interactions are switched on 23. Onsite interactions thus enhance the tendency 


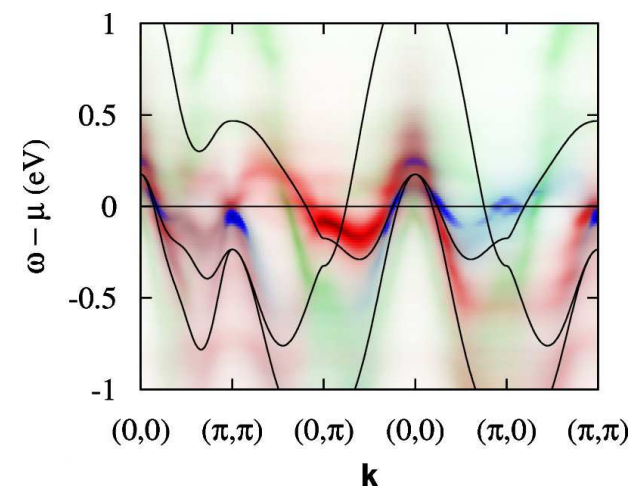

Figure 2. As figure 1 but without orbital energy splitting. Instead, there are anisotropic AFM couplings, see (8), acting within the directly solved four-site cluster, i.e., on a very short distance only. $J_{x}=0.04 \mathrm{eV} \gg J_{y}=0.01 \mathrm{eV}$

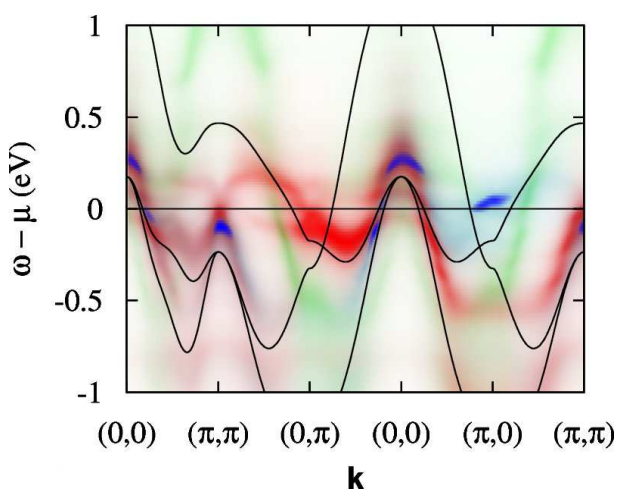

Figure 3. As figure 1 but without orbital energy splitting. Instead, there is a phenomenological difference in hopping parameter $t_{2}$ : it is $10 \%$ larger along $y$-direction.

to short-range magnetic correlations but do not appear to strengthen tendencies to a direct orbital energy splitting in a comparable manner. We did not find spontaneous symmetry breaking between the $x z$ and $y z$ orbitals, which can be investigated in the variational cluster-perturbation theory [32, 33].

Figure 3 illustrates an alternative way to induce an anisotropy: The hopping parameter $t_{2}$ is chosen $10 \%$ larger along the $y$-direction, a rather larger hopping anisotropy. For non-interacting electrons (not shown), this simply increases the dispersion somewhat, but does not raise the states at $Y$. As can be seen in figure 3 the interplay of the hopping anisotropy with onsite interactions, which favour a $(\pi, 0)$ or $(0, \pi)$ SDW, distorts the bands: The $y z$ band going from $\Gamma$ to $X$ has become very incoherent, and the only remaining coherent states at $X$ are above the Fermi level. It should be noted that the effect of an anisotropy on various hopping parameters is not consistent: It is largest for $t_{2}$, which is the larger NN hopping entering the kinetic energy of the $x z$ and $y z$ orbitals, see (2). $10 \%$ anisotropy of the other NN hoppings $t_{1}, t_{5}$ and $t_{7}$ leads to far smaller effects (not shown).

Finally, we take a closer look at the short-range magnetic interactions that were 
shown to induce band anisotropies in [23], where the strength of the AFM (along $x$ ) and FM (along $y$ ) couplings were chosen with opposite sign, but of equal strength. By varying the two parameters independently, we found that it is not necessary to have one FM and one AFM direction. For example, figure 2 shows a spectrum obtained for a case where both couplings are AFM, but the one along $x$ is much stronger $\left(J_{x}=0.04 \mathrm{eV}\right)$ than the one along $y\left(J_{y}=0.01 \mathrm{eV}\right)$. The AFM couplings act only within the directly solved cluster, i.e., they favour AFM bonds more along $x$ than along $y$. The clusters are coupled within cluster-perturbation theory only via the kinetic energy, i.e., there is no long-range magnetic order. The anisotropic signatures seen in the spectral density are comparable to those found for $J_{x}=-J_{y}=0.015 \mathrm{eV}[23]$. As the order parameter for nematic order is of higher order, its spontaneous symmetry breaking can not be studied in cluster-perturbation theory.

\section{Discussion and Conclusions}

We investigated how various mechanisms of breaking the four-fold lattice symmetry of a three-orbital model for Fe-As planes manifest themselves in the spectral density. Onsite interactions bring the system here close to a SDW transition, where shortrange magnetic couplings have previously been found to be more effective at breaking rotational symmetry than in the non-interacting system. We find that an orbital energy splitting and anisotropic hoppings can lead to qualitatively similar features in the interacting bands as short-range magnetic correlations: the states at $X$ can be found at higher energies than those at $Y$ and can even move up to the Fermi level, see also previous studies [14, 23]. The band/orbital anisotropy near the Fermi surface can be similarly pronounced both in cases with a strong total orbital polarization $n_{x z}-n_{y z} \approx 0.38$ (found for $\Delta=0.1 \mathrm{eV}$ as in figure 1) and with nearly vanishing polarization $n_{x z}-n_{y z} \approx 0.02$ (found for the anisotropic AFM couplings as in figure2). A splitting of $\approx 100 \mathrm{meV}$ between the $x z$ at $Y$ and the $y z$ states at $X$, which moves the latter close to or just above the chemical potential, can be induced by (i) an orbital energy splitting of $\Delta=0.1 \mathrm{eV}$, (ii) $10 \%$ anisotropy in the hopping parameter $t_{2}$ and (iii) a magnetic anisotropy of $30 \mathrm{meV}$ between the $x$ and $y$ directions.

There are differences in the results obtained in the three scenarios: In the case of an orbital splitting, the changes around $\Gamma=(0,0)$ are more pronounced than in either the magnetic scenario of the scenario with anisotropic hopping. In the latter case, the spectra appear less coherent even near the Fermi level, and the $y z$ band going from $\Gamma$ to $X$ almost disappears except for states very close to $X$ that are above the Fermi level. Comparing to ARPES [7, 8, 9, 10, this lack of coherence does not appear to be in good agreement. Distinction between the other two scenarios is more difficult and has previously been discussed for an orbital energy difference in the non-interacting model [23]. The conclusions drawn there remain valid for the interacting model: ARPES data look more consistent with slighter changes around $\Gamma$ than those arising from an orbital energy difference large enough to raise the states at $X$ to the Fermi level. However, it has to be stressed that both our model (including only the three most important orbitals) and our method (where the impact of onsite correlations is only treated exactly within a very small four-site cluster) imply substantial approximations. The most robust conclusion to be drawn might thus be that a decision between the scenarios based on experimental ARPES data remains difficult. Finally, it is also thinkable that different mechanisms are driving the breaking of the fourfold lattice symmetry in various compounds of the pnictide family. 
Breaking of four-fold lattice symmetry in a model for pnictide superconductors

\section{Acknowledgments}

This research was supported by the DFG under the Emmy-Noether programme and via the Graduate Training Group GRK 1621.

\section{References}

[1] Johnston D 2010 Adv. in Phys., 59 803-1061

[2] Paglione J and Greene R L 2010 Nature Physics, 6645

[3] Boeri L, Dolgov O V, and Golubov A A 2008 Phys. Rev. Lett. 101026403

[4] Chubukov A 2012 Annual Review of Condensed Matter Physics 3 57-92

[5] Chu J-H, Analytis J G, De Greve K, McMahon P L, Islam Z, Yamamoto Y, and Fisher I R 2010 Science $\mathbf{3 2 9} 824$

[6] Dusza A, Lucarelli A, Pfuner F, Chu J, Fisher I R, and Degiorgi L 2011 EPL 9337002

[7] Yi M, Lu D H, Chu J H, Analytis J G, Sorini A P, Kemper A F, Mo S-K, Moore R G, Hashimoto M, Lee W S, Hussain Z, Devereaux T P, Fisher I R, and Shen Z-X 2011 PNAS 1086878

[8] Yi M, Lu D H, Moore R G, Kihou K, Lee C-H, Iyo A, Eisaki H, Yoshida T, Fujimori A, and Shen Z-X 2011 arXiv:1111.6134

[9] He C, Zhang Y,. Xie B P, Wang X F, Yang L X, Zhou B, Chen F, Arita M, Shimada K, Namatame H, Taniguchi M, Chen X H, Hu J P, and Feng D L 2010 Phys. Rev. Lett. 105 117002

[10] Zhang Y, He C, Ye Z R, Jiang J, Chen F, Xu M, Ge Q Q, Xie B P, Wei J, Aeschlimann M, Cui X Y, Shi M, Hu J P, and Feng D L 2012 Phys. Rev. B 85085121

[11] de la Cruz C, Huang Q, Lynn J W , Li J, Ratcliff W II, Zarestky J L, Mook H A, Chen G F, Luo J L, Wang N L, and Dai P 2008 Nature 453 899-902

[12] Krüger F, Kumar S, Zaanen J, and van den Brink J 2009 Phys. Rev. B 79054504

[13] Lv W, Krüger F, and Phillips P 2010 Phys. Rev. B 82045125

[14] Lv W and Phillips P 2011 Phys. Rev. B 84174512

[15] Fernandes R M, Abrahams E, and Schmalian J 2011 Phys. Rev. Lett., 107217002

[16] Fernandes R M, Chubukov A V, Knolle J, Eremin I, and Schmalian J 2012 Phys. Rev. B 85 024534

[17] Fang C, Yao H, Tsai W F, Hu J P, and Kivelson S A 2008 Phys. Rev. B 77224509

[18] Xu C, Müller M, and Sachdev S 2008 Phys. Rev. B 78020501

[19] Cano A, Civelli M, Eremin I, and Paul I 2010 Phys. Rev. B 82020408

[20] Liang S, Alvarez G, Şen C, Moreo A, and Dagotto E 2011 arXiv:1111.6994

[21] Brydon P M R and Timm C 2009 Phys. Rev. B 79180504

[22] Paul I 2011 Phys. Rev. Lett. 107047004

[23] Daghofer M, Nicholson A, and Moreo A 2012 arXiv:1202.3656

[24] Daghofer M, Nicholson A, Moreo A, and Dagotto E R 2010 Phys. Rev. B 81014511

[25] Lee P A and Wen X G 2008 Phys. Rev. B 78144517

[26] Eschrig H and Koepernik K 2009 Phys. Rev. B 80104503

[27] Lee S H, Xu G, W Ku, Wen J, Lee C, Katayama N, Xu Z, Ji S, Lin Z, Gu G, Yang H-B, Johnson P, Pan Z-H, Valla T, Fujita M, Sato T, Chang S, Yamada K, and Tranquada J 2010 Phys. Rev. B, 81220502

[28] Castellani C, Natoli C R, and Ranninger J 1978 Phys. Rev. B 18 4945-4966

[29] Oleś A M 1983 Phys. Rev. B 28 327-339

[30] Gros C and Valentí R 1993 Phys. Rev. B 48 418-425

[31] Sénéchal D, Perez D, and Pioro-Ladrière M 2000 Phys. Rev. Lett. 84 522-525

[32] Potthoff M, Aichhorn M, and Dahnken C 2003 Phys. Rev. Lett. 91206402

[33] Dahnken C, Aichhorn M, Hanke W, Arrigoni E, and Potthoff M 2004 Phys. Rev. B 70245110

[34] Daghofer M, Moreo A, Riera J A, Arrigoni E, Scalapino D J, and Dagotto E R 2008 Phys. Rev. Lett. 101237004

[35] Yu R, Trinh K T, Moreo A, Daghofer M, Riera J A, Haas S, and Dagotto E R 2009 Phys. Rev. B 79104510 